\title{
Viroses de l'ail: I. - Mise en évidence et essais de caractérisation par immunoélectromicroscopie d'un complexe de trois virus chez différentes populations d'ail atteintes de mosaïque
}

\author{
Brigitte DELECOLLE \& Hervé LOT \\ I.N.R.A., Station de Pathologie végétale, Centre de Recherches d'Avignon, F 84140 Montfavet
}

\begin{abstract}
RÉSUMÉ
Ail,

Virus,

Bigarrure de l'oignon, Identification, Immunoélectromicroscopie.
\end{abstract}

\begin{abstract}
L'utilisation combinée de différentes méthodes montre que l'ail atteint de mosaïque grave (ail précoce type "Blanc de la Drôme ") ou de mosaïque atténuée (ail tardif rose type "Fructidor ») est porteur de trois virus différents: un virus appartenant au groupe Carlavirus et deux virus appartenant au groupe Potyvirus. Un de ces deux potyvirus a été identifié par immunoélectromicroscopie : il est identique sérologiquement au virus de la bigarrure de l'oignon (O.Y.D.V.). C'est ce virus qui est responsable des symptômes de mosaïque. En revanche, l'autre potyvirus et le carlavirus ne provoquent pas de symptôme. En effet, ce complexe " avirulent» de deux virus a été retrouvé chez tous les ails type «Blanc de la Drôme » sans symptôme, issus de sélection clonale tels que «Messidrôme » et «Thermidrôme». Ces clones sont donc «porteurs sains » de deux virus, qui pour l'instant, n'ont pu être identifiés.

On peut reproduire facilement, sur des ails «porteurs sains», par voie mécanique, les symptômes de mosaïque en leur inoculant un extrait d'ail malade (soit d'ail blanc, soit d'ail rose) : on constate alors que ces ails « portcurs sains" ont acquis l'O.Y.D.V. et qu'ils présentent alors des symptômes de mosaïque grave.
\end{abstract}

\author{
SUMMARY \\ Garlic, \\ Viruses, \\ Onion yellow dwarf, \\ Identification, \\ Immune electron micros- \\ copy.
}

Garlic viruses: detection and partial characterization with immune electron microscopy of three viruses in different garlic populations with mosaic

The combined use of different methods shows that garlic with a severe mosaic (early garlic « Blanc de la Drôme" type) or garlic with a weak mosaic (late pink garlic «Fructidor » type) harbour three different viruses: one belongs to the Carlavirus group and the two others to the Potyvirus group.

One of these potyvirus has been identified by immunoelectromicroscopy as identical serologically to O.Y.D.V. (Onion Yellow Dwarf Virus) : it is the one responsible for the mosaic symptoms expression. On the other hand, the second potyvirus and the carlavirus do not induce symptoms. This avirulent complex infects all the symptomless garlic of the "Blanc de la Drôme" clones issued from clonal selection as "Messidrôme" and «Thermidrôme ». These clones are therefore symptomless carriers of two viruses which are not yet identified.

Symptoms of mosaic can be reproduced on symptomless carriers garlic plants by mechanical inoculation of diseased garlic extracts from early or late pink garlics : it is proved that such inoculated plants harbour then O.Y.D.V. and develop severe mosaic. Similar results were obtained with true healthy garlic plants issued from tip culture and controlled as virus free.

\section{INTRODUCTION}

Reconnues dès 1946 par BRIERLEY \& SMITH, puis par MESSIAEN \& ARNOUX en 1960, les viroses de l'ail ont fait l'objet de nombreux travaux. L'hypothèse d'un seul virus pathogène, le virus de la mosaïque de l'ail (G.M.V.) a longtemps prévalu (MESSIAEN et al., 1965) mais des études cytologiques réalisées en 1976 (CADILHAC et al.) ont révélé la présence de 2 virus : un virus de type Carlavirus assimilé au G.M.V. et un virus du groupe Potyvirus non identifié.

On avait alors supposé que les symptômes de mosaïque sévère observés dans certaines populations d'ail pouvaient être dus à la présence du complexe de ces 2 virus, les symptômes de mosaïque atténuée pouvant être liés, soit à la présence de l'un de ces 2 virus, soit à l'existence de souches particulières.

En réalité, l'étiologie de ces maladies s'est révélée beaucoup plus complexe et nous avons donc entrepris d'étudier les populations virales se trouvant dans des ails d'origines diverses (ails issus de sélection sanitaire, sans symptôme apparent, ails atteints de mosaïque grave et de mosaïque faible), afin de préciser la relation entre la population virale et les symptômes observés. 


\section{MATÉRIEL ET TECHNIQUES}

\section{A. Matériel végétal}

Nous avons étudić les clones d'ail suivants :

1) Ail « Blanc de la Drôme » avec les symptômes graves et typiques de la maladie : mosaïque striée jaunc, jeunes feuilles déformées et filiformes, réduction de croissance importante ;

2) Ail «Violet de Cadours" présentant les mêmes types de symptômes que ceux décrits ci-dessus ;

3) Ail «Blanc de la Drôme" ayant fait l'objet de sélection sanitaire sévère (sélection clonale, contrôles rigoureux en cours de culture) et ne présentant aucun symptôme de la maladie : clones « Messidrôme » et «Thermidrôme ";

4) Ails roses tardifs tels que "Fructidor", "Brignoles 4 » et un ail rose « Roumain» présentant des symptômes de mosaique verte atténuée et dont la croissance est moins affectée que dans le cas du "Blanc de la Drôme ";

5) Ails roses issus de culture d'apex ne présentant aucun symptôme : clone «Moulinen » et « Fructidor 20 ».

Les souches de virus de bigarrure de l'oignon (O.Y.D.V.), de virus latent de l'échalote (S.L.V.) et de virus de striure du poireau (L.Y.S.V.) ont été isolées à la station à partir d'oignon, d'échalote et de poireau, respectivement.

\section{B. Transmission mécanique}

Les extraits infectieux sont préparés à température ambiante par broyage d' $1 \mathrm{~g}$ de feuille dans $4 \mathrm{ml}$ de tampon phosphate $0,06 \mathrm{M}, \mathrm{pH} \mathrm{7,0}$ auquel on a ajouté 1 p. $100 \mathrm{de}$ pectinase et $300 \mathrm{mg}$ de carborundum. Les inoculations sont réalisées sur des plants cultivés en serre ayant 2 feuilles bien développées (15 $\mathrm{cm}$ de longueur environ). On inocule les 2 feuilles en les frottant vigoureusement sur toute leur longueur.

\section{Coloration négative et histogramme}

Les virus sont extraits par broyage de $5 \mathrm{~mm}^{2}$ environ de feuilles dans une goutte d'eau distillée contenant 1 p. 100 de pectinase. L'extrait obtenu est déposé sur une grille recouverte d'un film de formvar et coloré par addition d'une goutte d'acide phosphotungstique à 1 p. 100 pH 7,0.

Les mesures des longueurs des virus ont été réalisées sur les négatifs à l'aide d'une loupe monoculaire grossissant 10 fois, munie d'un micromètre.

Avant d'effectuer les prises de vue au microscope électronique (Philips E.M. 301), on a utilisé un standard externe de calibration afin d'obtenir de façon précise le grossissement du microscope.

\section{Cytologie}

Les échantillons (fragments de feuille de $2 \mathrm{~mm}^{2}$ environ) sont fixés à froid pendant $4 \mathrm{~h}$ dans du glutaraldéhyde à 4 p. 100 pH 7,2 tamponné avec du cacodylate de sodium $0,1 \mathrm{M}$. Ils sont ensuite rincés dans 4 bains, de $1 \mathrm{~h}$ chacun, de tampon cacodylate de sodium $0,1 \mathrm{M}$ pH 7,2 auquel on a ajouté 3 p. 100 de glucose. Enfin ils sont post-fixés pendant $1 \mathrm{~h} 30$ dans du tétroxyde d'osmium à 1 p. 100 dans un tampon cacodylate à $0,1+3$ p. 100 glucose.

Après rinçage dans 5 bains d'eau bidistillée, les échantillons sont déshydratés à l'acétone et inclus dans de l'araldite $M$ suivant les procédés classiques.
Les coupes ultrafines sont contrastées avec du permanganate de potassium à 1 p. 100 .

\section{E. Purification du complexe viral et obtention d'un sérum polyvalent}

La purification est réalisée à partir d'ail «Blanc de la Drôme " chroniquement infecté et présentant une mosaïque grave. Les feuilles ( $200 \mathrm{~g}$ environ) sont broyées dans $300 \mathrm{ml}$ de citrate trisodique $0,3 \mathrm{M}$ pH 8,5 contenant 0,3 p. 100 de mercaptoéthanol et 1 p. 100 de pectinase. Après filtration sur étamine, les fibres retenues sont réextraites en présence de $200 \mathrm{ml}$ du même tampon. Le filtrat est alors incubé $20 \mathrm{mn}$ à température ambiante puis agité pendant une nuit à $4{ }^{\circ} \mathrm{C}$ en présence de butanol à 8 p. 100 . Après clarification, le surnageant, filtré sur coton de verre, est ultracentrifugé $2 \mathrm{~h}$ à $78000 \mathrm{~g}$. Les culots sont repris dans du citrate trisodique $0,02 \mathrm{M}$-phosphate disodique $0,002 \mathrm{M}$ pH 8,5 contenant 1 p. 100 de Triton $X-100$; la suspension est déposée sur coussin de saccharose 20 p. 100 dissous dans le tampon de reprise des culots et ultracentrifugé $3 \mathrm{~h}$ à $78000 \mathrm{~g}$. Le culot est repris dans $2 \mathrm{ml}$ de citrate-phosphate pH 8,5. 0,2 p. 100 de formol est ajouté à cette préparation qui est utilisée alors pour 3 injections à un lapin.

Les injections de suspension virale additionnée d'adjuvant incomplet de Freund (v/v) sont réalisées à une semaine d'intervalle par voie intramusculaire. Un sérum de titre satisfaisant est obtenu après la $8^{\mathrm{c}}$ injection: c'est l'antisérum «B.D.V.» (Blanc de la Drôme virosé) contenant des anticorps vis-à-vis des 3 virus présents dans le complexe.

\section{F. Immunoélectromicroscopie (I.E.M.)}

Nous utilisons les sérums suivants :

- antisérum O.Y.D.V. (Onion Yellow Dwarf Virus) titre: $1 / 1024$

- antisérum L.Y.S.V. (Leek Yellow Stripe Virus), titre : $1 / 1024$

— antisérum S.L.V. (Shallot Latent Virus), titre : 1/512.

Ces trois sérums ont été aimablement fournis par MM. BOS \& MAAT (Hollande).

- antisérum «B.D.V. » qui réagit en I.E.M. avec certains virus du complexe viral jusqu'à la dilution : 1/4 096.

\section{1) Préparation des extraits}

Ils sont préparés par broyage au 1/5 dans du tampon phosphate Sörensen $0,06 \mathrm{M}$ pH 7,0 auquel on a ajouté 1 p. 100 de pectinase. Le broyat obtenu est clarifié par une centrifugation de $10 \mathrm{mn}$ à $5000 \mathrm{~g}$.

\section{2) Méthode de la "décoration"}

Les techniques utilisées sont celles décrites par MILNE \& LUISONI (1975) modifiées par ROBERTS (comm. pers.).

Les sérums sont utilisés à différentes dilutions : 1/8, 1/16, $1 / 32,1 / 64,1 / 128,1 / 256,1 / 512,1 / 1024$; à $20 \mu l$ de sérum sont ajoutés $10 \mu \mathrm{l}$ d'extrait clarifié, dilué de façon à n'avoir que 5 à 10 particules virales par champ au grossissement $\times 22000$. Cela évite d'avoir, après réaction sérologique, un phénomène d'agrégation des particules virales. Suivant les cas, nous avons dû diluer les extraits au $1 / 10$ ou jusqu'au $1 / 250$. Le mélange anticorps-antigène est alors incubé à $37^{\circ} \mathrm{C}$ pendant $30 \mathrm{mn}$. 
La réaction sérologique est visualisée au microscope électronique après coloration au molybdate d'ammonium à 1 p. $100 \mathrm{pH} \mathrm{7,0.}$

\section{3) Méthode de Derrick}

La méthode de DERRICK (1973) est utilisée avec les antisérums O.Y.D.V. et B.D.V. aux dilutions $1 / 1024$ et 1/4 096 respectivement. La fixation des anticorps sur les grilles est effectuée par incubation à $37^{\circ} \mathrm{C}$ pendant $3 \mathrm{~h}$. La fixation des antigènes (dilués de façon à n'avoir qu'1 à 2 particules virales par champ à $G \times 22000$ ) sur les anticorps s'est effectuée à $20^{\circ} \mathrm{C}$ pendant $3 \mathrm{~h}$.

\section{RÉSULTATS}

\section{A. Etude des populations virales dans des ails d'origines diverses}

1) Ail «Blanc de la Drôme » et Ail «Violet de Cadours» présentant des symptômes de grave mosaïque

\section{a) Morphologie des particules virales}

Les recherches effectuées sur la morphologie des virus contenus dans ces ails montrent qu'il existe au moins 2 catégories de particules virales appartenant à 2 groupes de virus bien distincts : les unes assez rigides et courtes, d'une longueur d'environ $600 \mathrm{~nm}$ correspondent au groupe des carlavirus ; les autres plus flexueuses, plus longues, d'une longueur d'environ $700 \mathrm{~nm}$ correspondent au groupe des potyvirus (fig. 1). Les histogrammes de la fréquence de la longueur de ces virus montrent l'existence de 2 pics principaux correspondant aux longueurs 600 et $700 \mathrm{~nm}$ (fig. 2).

\section{b) Cytologie}

Les études cytologiques confirment, chez ces ails, la présence de virus appartenant à ces 2 groupes. En effet, dans les cellules épidermiques et dans le mésophylle, on observe, soit la présence d'agrégats importants de virions dont l'aspect varie suivant le plan de coupe (faisceaux compacts de virions, amas globuleux ou spiralés) caractéristiques des virus du groupe carlavirus (fig. 3), soit la présence de virions épars dans le cytoplasme accompagnés de structures de type "pinwheels 》 caractéristiques des infections provoquées par des virus du groupe potyvirus (fig. 4).

\section{c) Immunoélectromicroscopie}

L'enrobage des particules virales (méthode de la décoration) d'un extrait d'oignon infecté par l'O.Y.D.V. avec les anticorps spécifiques (antisérum O.Y.D.V.) s'observe aisément jusqu'à la dilution 1/512.

Lorsqu'on fait réagir un extrait d'ail malade avec ce même antisérum, on constate qu'une partie des virus de cet extrait a fortement réagi avec le sérum jusqu'à une dilution 1/256, la dilution 1/1024 étant la limite pour une observation aisée. Les anticorps se sont fixés sur la particule virale donnant à celle-ci un aspect granuleux et plus épais (fig. 5), alors que les virus qui n'ont pas réagi ont gardé leur aspect lisse et fin tel qu'on peut l'observer habituellement en coloration négative.

Quand on fait réagir un extrait d'ail malade avec l'antisérum B.D.V., on observe une décoration en 3 temps des particules virales de l'extrait : jusqu'à la dilution du sérum de $1 / 128$ tous les virus sont décorés (fig. 6) ; à partir de la dilution $1 / 256$ et jusqu'au $1 / 1024$, on trouve des virus fortement décorés, des virus faiblement décorés et des particules entièrement lisses. Enfin, à la dilution 1/2048 et 1/4 096, seul un petit nombre de virus est décoré (fig. 7).

On peut penser que l'antisérum B.D.V. contient des anticorps spécifiques de 3 virus présents dans les préparations purifiées à partir de «Blanc de la Drôme " atteint de mosaïque et que ces anticorps réagissent à certaines dilutions en fonction de leur concentration dans l'antisérum polyvalent.

La présence du $2^{\mathrm{e}}$ virus flexueux chez l'ail malade est confirmée par l'étude de l'histogramme de fréquence de la longueur des virus transmis d'ail malade à Chenopodium quinoa: les histogrammes montrent la présence de 2 pics correspondant à un carlavirus et à un potyvirus. Or, les contrôles réalisés par I.E.M. montrent que l'O.Y.D.V. de l'ail ne passe pas sur Chenopodium quinoa. C'est donc que chez l'ail malade, outre l'O.Y.D.V. et le carlavirus, il existe

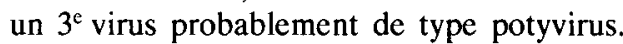

Chez l'ail malade, il existe ainsi un potyvirus sérologiquement identique à l'O.Y.D.V., un carlavirus et probablement un autre virus flexueux.

Les tentatives de caractérisation de ces virus en utilisant les antisérums S.L.V. et L.Y.S.V. n'ont pas donné de réaction positive bien que ces 2 sérums réagissent fortement avec nos souches S.L.V. et L.Y.S.V. de référence (fig. 8 et fig. 9).

\section{2) Ails roses: "Fructidor", "Brignoles 4", "Ail Rou- main", présentant une mosaïque atténuée}

La même série de contrôles a été réalisée ; les histogrammes de fréquence de la longueur des virus ont montré qu'il existait 2 classes de particules de type carlavirus et potyvirus; la cytologie a révélé la présence de "pinwheels» caractéristiques des potyvirus et d'amas de virions caractéristiques des carlavirus.

L'immunoélectromicroscopie enfin a permis de mettre en évidence chez tous ces ails, la présence d'O.Y.D.V.

Comme dans le cas des ails blancs, il n'a pas été possible de caractériser sérologiquement le carlavirus et le $2^{\mathrm{c}}$ virus flexueux.

\section{3) Ails "Blanc de la Drôme " issus de sélection massale et clonale ("Thermidrôme » et "Messidrôme») et ne pré- sentant aucun symptôme de mosaïque}

Bien qu'ils soient dépourvus de tout symptôme, tous les ails étudiés renferment des particules virales (fig. 10). Une étude des histogrammes de fréquence des longueurs de ces virus révèle, comme dans les cas précédents, la présence de 2 pics correspondant à 2 classes de virus différentes : carlavirus et potyvirus (fig. 11). En revanche, il n'a pas été possible par immunoélectromicroscopie de détecter, dans ces ails, la présence d'O.Y.D.V. Ces ails sans symptôme, apparemment sains, renferment donc 2 virus : un carlavirus et un potyvirus.

Ces 2 virus, comme dans les cas précédents, n'ont pas pu être identifiés au S.L.V. ni au L.Y.S.V. ; ce sont probablement les mêmes que ceux présents chez l'ail atteint de mosaïque grave ou de mosaïque atténuée. En effet, si on fait réagir un extrait d'ail sans symptôme avec l'antisérum polyvalent B.D.V., on observe une décoration de toutes les particules virales (fig. 12) et ceci jusqu'à la dilution $1 / 256$; à partir de la dilution $1 / 512$, certaines particules ont une décoration moins dense et, à la dilution $1 / 2048$, on peut distinguer 2 types de particules bien individualisées: les unes lisses et les autres décorées par les anticorps (fig. 13). 

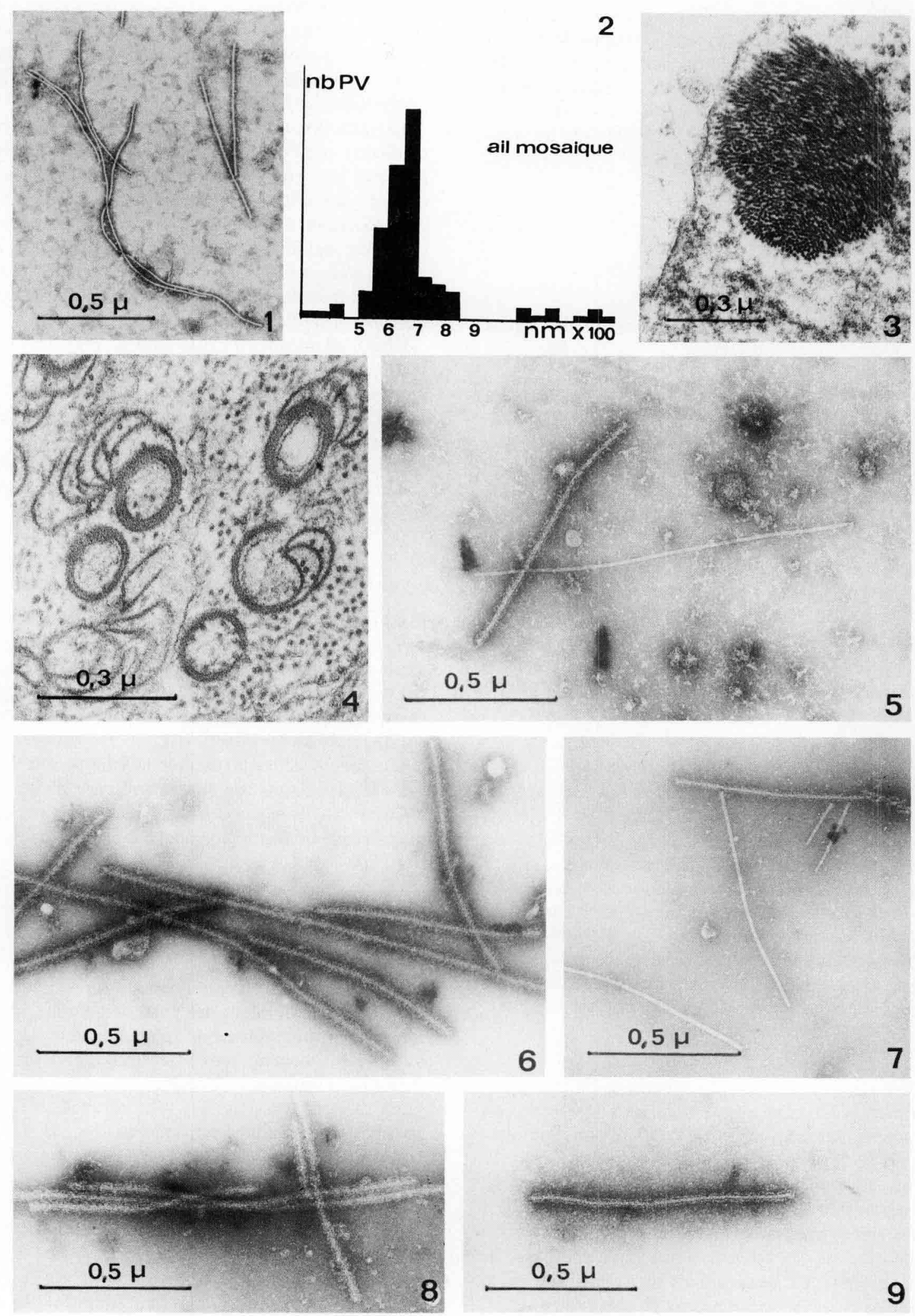

Planche 1 
Figure 1

Micrographie des particules virales d'un extrait d'ail "Blanc de la Drôme " infecté naturellement et présentant une mosaïque grave: noter la présence de virus flexueux de type potyvirus et de particules plus rigides de type carlavirus. $G \times 46000$.

Electron micrograph of viruses of naturally infected "Blanc de la Drôme "garlic showing a severe mosaic in a crude extract. Note flexuous rods of potyvirus group, shorter and less flexuous rods of carlavirus group. $G \times 46000$.

Figure 2

Histogramme de la fréquence des longueurs des virus présents dans ces extraits d'ails "Blanc de la Drôme " virosés (B.D.V.) : les 2 pics correspondent respectivement $\grave{a}$ la classe des carlavirus et des potyvirus.

Histogram of lengths "Blanc de la Drôme" garlic viruses with severe mosaic (B.D.V.) : 2 classes of viruses : carlavirus and potyvirus.

Figure 3

Cellule du mésophylle d'ail B.D.V. : structures de type pinwheels $(P W)$ caractéristiques des infections provoquées par des potyvirus. $G \times 94000$.

Mesophyll cell of diseased garlic (B.D.V.) : pinwheels-type structures are typically related with potyvirus group infections. $G \times 94000$.

Figure 4

Cellule du mésophylle d'ail B.D.V. : noter la présence d'amas de virions en formation compacte caractéristique des carlavirus. $G \times 68000$.

Mesophyll cell of diseased garlic (B.D.V.): note the presence of bundles of virus particles related with carlavirus group infections. $G \times 68000$.

Figure 5

Immunoélectromicroscopie: réaction sérologique entre un extrait d'ail B.D.V. et un sérum anti O.Y.D.V. à la dilution 1/128: les anticorps se sont fixés sur certaines particules virales leur donnant ainsi un aspect plus épais et rugueux tandis que les virus qui n'ont aucune relation sérologique avec l'O.Y.D.V. gardent leur aspect lisse et fin. $G \times 60000$.

Immune electron microscopy: garlic viruses of crude extract from B.D.V. treated with antiserum O.Y.D.V. diluted 1:128. The antibody molecules are attached to some virus particles when unrelated viruses are not coated. $G \times 60000$

\section{Figure 6}

Immunoélectromicroscopie: réaction sérologique entre un extrai d'échalote et le sérum anti S.L.V. (Shallot Latent Virus) à la dilution 1/64. $G \times 60000$.

Immune electron microscopy: shallot extract treated with antiserum S.L.V. diluted $1: 64$. $G \times 60000$.

Figure 7

Immunoélectromicroscopie : réaction sérologique entre un extrait de poireau et le sérum anti L.Y.S.V. (Leek Yellow Stripe Virus) à la dilution $1 / 128 . G \times 60000$.

Immune electron microscopy: leek extract treated with antiserum L.Y.S.V. diluted $1: 128 . G \times 60000$.

Figure 8

Immunoélectromicroscopie: réaction sérologique entre un extrait d'ail B.D.V. et son sérum homologue à la dilution 1/128: toutes les particules virales sont décorées. $G \times 60000$.

Immune electron microscopy: garlic B.D.V. extract treated with antiserum B.D.V. diluted $1: 128$ all the virus particles are coated. $G \times 60000$

Figure 9

Immunoélectromicroscopie : réaction sérologique entre un extrait d'ail B.D.V. et son sérum homologue à la dilution 1/2048: seul un petit nombre de virus est encore décoré. $G \times 60000$.

Immune electron microscopy: garlic B.D.V. extract treated with antiserum B.D.V. diluted $I: 2048-\mathrm{Few}$ particles are still coated. $G \times 60000$.
4) Ails roses issus de culture d'apex ("Moulinen" et "Fructidor 20 ") sans aucun symptôme

La méthode de Derrick appliquée à des extraits de ces ails ne présentant aucun symptôme, a révélé que :

- certaines plantes étaient absolument indemnes de virus ;

- d'autres plantes renfermaient deux types de virus : un Carlavirus et un Potyvirus; dans ce cas, l'application de la méthode de décoration avec l'antisérum O.Y.D.V. a mis en évidence qu'il ne s'agissait pas d'O.Y.D.V.

\section{B. Rôle de l'O.Y.D.V. dans l'extériorisation des symptô- mes de mosaique}

Nous avons inoculé l'ail "Messidrôme " sans symptôme soit avec un extrait de «Blanc de la Drôme», soit avec un extrait d'ail rose «Brignoles 4 », tous 2 présentant des symptômes de mosaïque.

Dans les 2 cas, les plantes inoculées ont présenté une légère striure à la base des feuilles; un mois après, la mosaïque s'aggrave et se généralise et on note une réduction de croissance importante tandis que les jeunes feuilles sont déformées et filiformes. Les contrôles réalisés en I.E.M. révèlent alors la présence d'O.Y.D.V. dans les extraits réalisés à partir des « Messidrômes » inoculés.

L'O.Y.D.V. peut être détecté par cette méthode avant même l'apparition des symptômes, c'est-à-dire entre 8 et $15 \mathrm{j}$ après l'inoculation.

\section{DISCUSSION}

Le tableau 1 résume nos résultats d'essais de caractérisation des virus dans les ails naturellement infectés ou inoculés :

- l'ail présentant des symptômes de mosaïque grave ou atténuée est infecté par un potyvirus sérologiquement identique à l'O.Y.D.V. et par 2 autres virus - un carlavirus et un potyvirus - actuellement non caractérisés. Ces 2 derniers infectent également des ails apparemment sains : il s'agit d'un complexe avirulent.

De ces études il ressort que l'ail « Blanc de la Drôme», extériorise de graves symptômes chaque fois qu'il est infecté par l'O.Y.D.V. Par contre, les ails roses de type « Fructidor" ne présentent avec ce virus que des symptômes de mosaïque atténuée. Les différences de symptômes observées sur ails blancs et roses semblent être davantage liées au caractère propre de la variété d'ail plutôt qu'à l'existence de souches d'O.Y.D.V. plus ou moins agressives.

Les 2 autres virus, présents chez les ails malades ou apparemment sains, ne sont pas sérologiquement reliés aux autres virus décrits chez les Allium $s p$. Le carlavirus pourrait être désigné sous le nom de virus latent de l'ail (Garlic Latent Virus) par analogie avec le virus latent de l'échalote ; celui-ci a été trouvé en association avec 1'O.Y.D.V. dans l'échalote (Bos et al., 1978b ; DELECOLLE, résultats non publiés) et avec le L.Y.S.V. dans le poireau (Bos et al., 1978a ; PALUDAN, 1980). La caractérisation de ces 2 virus 

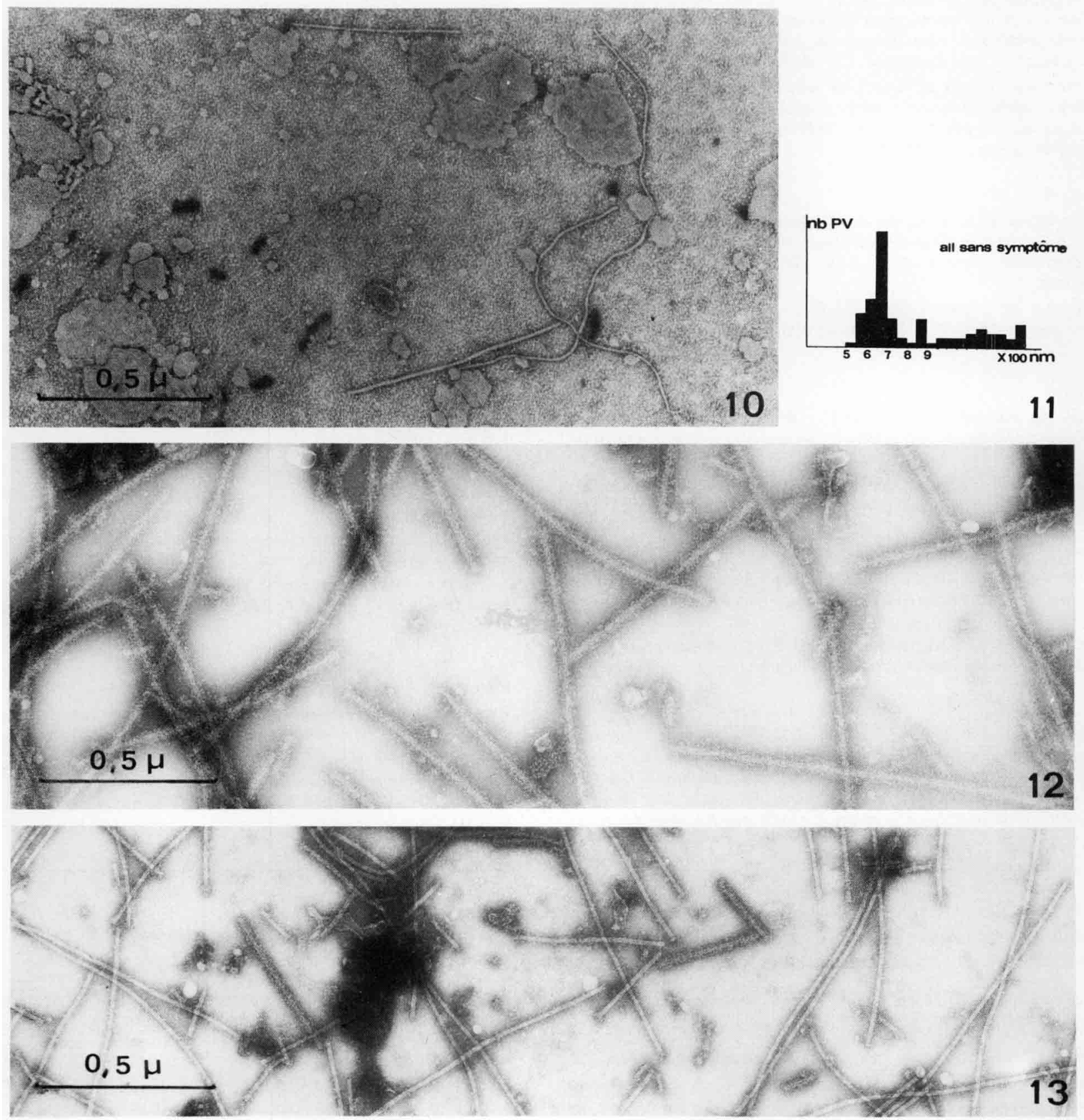

Planche 2

Figure 10

Micrographie des particules virales d'un extrait d'ail "Messidrôme » sans symptôme: noter la présence de particules flexueuses de type potyvirus et de particules plus rigides de type carlavirus. $G \times 46000$. Electron micrograph of crude extract of * Messidrôme " symptomless garlic: note the presence of long, flexuous particles (potyvirus group) and more slight and short particles (carlavirus group). $G \times 46000$

\section{Figure 11}

Histogramme de la fréquence des longueurs des virus présents dans des extraits d'ail "Messidrôme » sans symptôme; les 2 pics correspondent respectivement à la classe des carlavirus et des potyvirus. Histogram of lengths of viruses of symptomless "Messidrôme " garlic -2 classes : poty and carlavirus.
Figure 12

Immunoélectromicroscopie: réaction sérologique entre un extrait d'ail "Messidrôme" et le sérum anti B.D.V. à la dilution 1/64: toutes les particules virales sont décorées par les anticorps. $G \times 60000$.

Immune electron microscopy: "Messidrôme " garlic extract treated with antiserum B.D.V. diluted 1:64 - all the virus particles are coated. $G \times 60000$

Figure 13

Immunoélectromicroscopie: réaction sérologique entre un extrait d'ail "Messidrôme" et le sérum anti B.D.V. à la dilution 1/2048: seul un petit nombre de virus est décoré. Dans l'ail sans symptôme il y a bien 2 types de particules différentes. $G \times 60000$.

Immune electron microscopy: garlic "Messidrôme " extract treated with antiserum B.D.V. diluted $1: 2048$ - only a few particles are coated: in symptomless garlic there are 2 different types of viruses. $G \times 60000$. 
TABLEAU 1

Populations virales d'ails d'origines diverses

Viral populations of different garlic clones

\begin{tabular}{|c|c|c|c|c|}
\hline Ails & Cartavirus & Potyvirus $\mathrm{x}$ ? & O.Y.D.V. & Symptômes \\
\hline Blanc de la Drôme non régénéré (B.D.V.) & + & + & + & \multirow{3}{*}{ Mosaïque grave } \\
\hline Violet de Cadours & + & + & + & \\
\hline Fructidor non régénéré & + & + & + & \\
\hline Brignoles 4 & + & + & + & \multirow[t]{2}{*}{ Mosaïque atténuée } \\
\hline Roumain & + & + & + & \\
\hline Messidrôme & + & + & - & \multirow{2}{*}{ Pas de symptôme } \\
\hline Thermidrôme & + & + & - & \\
\hline Messidrôme inoculé avec B.D.V. & + & + & + & \multirow{3}{*}{$\begin{array}{l}\text { Mosaïque grave } \\
\text { Inoculation } \\
\text { réussie à } 100 \text { p. } 100 \\
\text { Pas de symptôme }\end{array}$} \\
\hline Messidrôme inoculé avec Brignoles 4 & + & + & + & \\
\hline Fructidor Méristème & - & - & - & \\
\hline
\end{tabular}

de l'ail par leurs propriétés biologiques, sérologiques et physicochimiques est entreprise. Leur effet sur les rendements d'une culture d'ail va être également étudié puisque nous disposons maintenant de clones totalement indemnes de virus.

L'étude des caractéristiques des souches O.Y.D.V. infectant l'ail se poursuit. S'il est possible de réussir l'infection d'oignons en inoculant un extrait de "Blanc de la Drôme » virosé et de montrer par I.E.M. que les symptômes observés sont liés à la présence de l'O.Y.D.V., les essais de transmission à l'ail avec la souche d'O.Y.D.V. isolée d'oignon se sont révélés, par contre, négatifs. Il s'agira de démontrer si cette impossibilité est liée à la présence d'inhibiteurs d'infection dans les extraits d'ail ou à une différence de l'aptitude pathogène de ces souches. La réussite de l'inoculation d'ail sain par une souche d'O.Y.D.V. pourra démontrer si ce virus est le seul responsable des symptômes de mosaïque. Nos résultats montrent que ces symptômes ne peuvent apparaître en l'absence de ce virus mais il n'est pas encore possible d'exclure totalement l'hypothèse selon laquelle la mosaïque résulterait d'une interaction entre l'O.Y.D.V. et l'un des 2 autres virus mis en évidence chez les plantes sans symptôme.

La contamination d'ails apparemment sains issus de cultures d'apex et de sélection sanitaire doit se faire essentiellement à partir de cultures d'ails malades, mais des cultures d'oignons ou d'échalotes virosés sont potentiellement des sources d'infection.

L'inventaire des espèces d'Allium spontanés porteurs d'O.Y.D.V. et des autres virus de l'ail qui a été entrepris aidera à mieux définir les foyers possibles d'infection.

Nos résultats montrent enfin l'intérêt de l'I.E.M. au moyen de la méthode de DERRICK (1973) pour détecter la présence éventuelle de virus chez l'ail. Il est désormais possible de réaliser chez des plants issus de cultures « in vitro » un dépistage précoce permettant d'éliminer les plants virosés et de ne garder, pour les multiplier, que les plants indemnes de virus.

Reçu le 15 février 1981. Accepté le 16 juin 1981.

\section{RÉFÉRENCES BIBLIOGRAPHIQUES}

Bos L., Huijberts N., Huttinga H., Maat D. Z., 1978a. Leek yellow stripe virus and its relationships to Onion Yellow Dwarf Virus; characterization, ecology and possible control. Neth. J. Plant Pathol., 84, 185-204.

Bos L., Huttinga H., Maat D. Z., 1978b. Shallot latent virus, a new carlavirus. Neth. J. Plant Pathol., 84, 227-237.

Brierley P., Smith F. F., 1946. Reaction of onion varieties to yellow dwarf virus and three similar viruses isolated from shallot, garlic and narcissus. Phytopathology, 36, 185-204.

Cadilhac Brigitte, Quiot J. B., Marrou J., Leroux J. P., 1976. Mise en évidence au microscope électronique de deux virus différents infectant l'ail (Allium sativum L.) et l'échalote (Allium cepa var. ascalonicum). Ann. Phytopathol., 8, 1, 65-72.

Derrick K. S., 1973. Quantitative assay for plant viruses using serologically specific electron microscopy. Virology, 56, 652-653.
Messiaen C. M., Arnoux M., 1960. Une maladie de l'ail probablement due à un virus ; son influence sur le rendement. Etudes de Virologie I.N.R.A., 29-30.

Messiaen C. M., Marrou J., 1965. Sélection sanitaire de l'ail : deux solutions possibles au problème de la mosaïque de l'ail : plantes sensibles saines ou plantes virosées tolérantes. C.R. $1^{e} J$. Phytiat. Phytopharm. circummediterr. Marseille, 204-207.

Milne R. G., Luisoni E., 1977. Rapid immune electron microscopy of virus preparations. in : K. Maramorosch \& H. Koprowski Methods in Virology, Academic Press, New-York, 6, 265-281.

Paludan N., 1980. Virusangreb i porrer : kortlaegning, diagnosticering, sortsmodtagelighedog overvintring. Saertryk Tidsskrift Planteavl, 84, 371-385. 